\title{
Kähler Geometry of Toric Varieties and Extremal Metrics
}

\author{
Miguel Abreu* \\ Institute for Advanced Study \\ revised version
}

February 7, 2008

1991 Mathematics Subject Classification: Primary 53C55, Secondary 14M25 $53 \mathrm{C} 25$ 58F05.

\begin{abstract}
A (symplectic) toric variety $X$, of real dimension $2 n$, is completely determined by its moment polytope $\Delta \subset \mathbb{R}^{n}$. Recently Guillemin gave an explicit combinatorial way of constructing "toric" Kähler metrics on $X$, using only data on $\Delta$. In this paper, differential geometric properties of these metrics are investigated using Guillemin's construction. In particular, a nice combinatorial formula for the scalar curvature $R$ is given, and the Euler-Lagrange condition for such "toric" metrics being extremal (in the sense of Calabi) is proven to be $R$ being an affine function on $\Delta \subset \mathbb{R}^{n}$. A construction, due to Calabi, of a 1-parameter family of extremal Kähler metrics of non-constant scalar curvature on $\mathbb{C} P^{2} \sharp \overline{\mathbb{C P}}^{2}$ is recast very simply and explicitly using Guillemin's approach. Finally, we present a curious combinatorial identity for convex polytopes $\Delta \subset \mathbb{R}^{n}$ that follows from the well known relation between the total integral of the scalar curvature of a Kähler metric and the wedge product of the first Chern class of the underlying complex manifold with a suitable power of the Kähler class.
\end{abstract}

\section{Introduction}

Associated to every convex polytope $\Delta \subset \mathbb{R}^{n}$, satisfying some nondegeneracy and integrality conditions, there is a unique (modulo suitable equivalence relation) closed connected symplectic manifold $\left(X_{\Delta}, \omega\right)$ of dimension $2 n$ together

*Supported by NSF grant DMS 9304580 through the Institute for Advanced Study. Current address: Departamento de Matemática, Instituto Superior Técnico, Av. Rovisco Pais, 1096 Lisboa Codex, Portugal. E-mail: mabreu@math.ist.utl.pt 
with a Hamiltonian action of the $n$-torus $T^{n}$ such that the image of the corresponding moment map $\phi$ is precisely $\Delta$. The triple $\left(X_{\Delta}, \omega, \phi\right)$ is called a (symplectic) toric variety. It follows from the construction, due to Delzant [4], that $X_{\Delta}$ comes also equipped with an intrinsic $T^{n}$-invariant complex structure compatible with the intrinsic symplectic form. In other words, $X_{\Delta}$ has an intrinsic "toric" Kähler metric. In [6] Guillemin gave an explicit combinatorial formula, in terms of moment data alone, for the potential of this Kähler metric as a function on $\Delta$ (via $\phi)$.

It is natural to ask if these canonical toric metrics have any special differential geometric properties. One possibility would be a relation with extremal Kähler metrics, introduced by Calabi in [1] and [2]. These metrics are defined as critical points of the square of the $L^{2}$-norm of the scalar curvature, considered as a functional on the space of all Kähler metrics in a fixed Kähler class. The extremal Euler-Lagrange equation is equivalent to the gradient of the scalar curvature being an holomorphic vector field (see [1]), and these metrics are always invariant under a maximal compact subgroup of the group of holomorphic transformations of the underlying complex manifold (see [2]). This last property is the main reason to look for a relation between extremal and toric metrics, the later being also invariant under a big group of holomorphic transformations (the torus $T^{n}$ ).

In this paper we look into this question, following Guillemin's approach in [6]. It turns out that the scalar curvature $R$ of a toric metric on $X_{\Delta}$, as a function on $\Delta$, has a nice combinatorial formula, while the extremal condition is $R$ being an affine function on $\Delta \subset \mathbb{R}^{n}$. A simple analysis of some examples leads quickly to the conclusion that, except for the case of cartesian products of complex projective spaces, the canonical toric metric does not satisfy the extremal condition. However Guillemin's construction not only gives an explicit combinatorial formula for the potential of the canonical toric metric, but can also be used to explicitly construct other toric Kähler metrics on $X_{\Delta}$ from data on $\Delta$ alone. One can try in this way to construct toric metrics satisfying the extremal condition. Although I do not know of a general procedure to do so, one can analyse by "hand" some simple yet non-trivial examples in real dimension 4 $(n=2)$. In particular a construction, due to Calabi [1], of a 1-parameter family of extremal metrics of non-constant scalar curvature on $\mathbb{C} P^{2} \sharp \overline{\mathbb{C}}^{2}$ can be recast very simply and explicitly using Guillemin's construction.

The paper is organized as follows. In $\S 2$ we give some very brief preliminaries on (symplectic) toric varieties. For more details on this beautiful subject we recommend Guillemin's recent monograph [5]. In $\S 3$ we review Guillemin's main construction and result in [6] (also explained in detail in an Appendix in [5]). The combinatorial formula for the scalar curvature $R$ of a toric metric on $X_{\Delta}$ and the extremal Euler-Lagrange condition are derived in $\delta$. In $\delta$ 国 we present a combinatorial identity that follows from the well known relation between the total integral of the scalar curvature of a Kähler metric and the wedge product of the first Chern class of the underlying complex manifold with 
a suitable power of the Kähler class. Calabi's family of extremal metrics on $\mathbb{C} P^{2} \sharp \overline{\mathbb{C} P}^{2}$ is discussed in $\$$.

Acknowledgements: This work was carried out while I was a postdoctoral member of the School of Mathematics of the Institute for Advanced Study. I take this opportunity to thank the staff and faculty of the Institute for providing a wonderful environment, both for me and my family.

I also want to thank V.Guillemin for the interest and encouragement given to this work, and A.Cannas da Silva and S.Simanca for pointing out corrections to an earlier version of this paper.

\section{Preliminaries on (symplectic) toric varieties}

Let $(X, \omega)$ be a closed connected $2 n$-dimensional symplectic manifold, and let $\tau: T^{n} \rightarrow \operatorname{Diff}(X, \omega)$ be an effective Hamiltonian action of the standard $n$-torus. Let $\phi: X \rightarrow \mathbb{R}^{n}$ be its moment map. The image $\Delta$ of $\phi$ is a convex polytope, called the moment polytope.

Not every convex polytope in $\mathbb{R}^{n}$ is the moment polytope of some triple $(X, \omega, \phi)$. The following definition characterizes the ones that are.

Definition 2.1 A convex polytope $\Delta$ in $\mathbb{R}^{n}$ is Delzant if:

(1) there are $n$ edges meeting at each vertex $p$;

(2) the edges meeting at the vertex $p$ are rational, i.e. each edge is of the form $p+t v_{i}, 0 \leq t \leq \infty$, where $v_{i} \in \mathbb{Z}^{n} ;$

(3) the $v_{1}, \ldots, v_{n}$ in (2) can be chosen to be a basis of $\mathbb{Z}^{n}$.

In [4] Delzant associates to every Delzant polytope $\Delta \subset \mathbb{R}^{n}$ a closed connected symplectic manifold $X_{\Delta}$ of dimension $2 n$ together with a Hamiltonian $T^{n}$-action such that the image of the corresponding moment map is precisely $\Delta$. Moreover, he shows that this is a bijective correspondence. More precisely, he proves the following:

Theorem 2.2 Let $(X, \omega)$ be a compact, connected, $2 n$-dimensional Hamiltonian $T^{n}$-space, on which the action of $T^{n}$ is effective with moment map $\phi$ : $X \rightarrow \mathbb{R}^{n}$. Then the image $\Delta$ of $\phi$ is a Delzant polytope, and $X$ is isomorphic as a Hamiltonian $T^{n}$-space to $X_{\Delta}$.

\section{The intrinsic Kähler metric on a (symplectic) toric variety}

It follows from Delzant's construction that $X_{\Delta}$ (or, because of Theorem 2.2, any effective hamiltonian $T^{n}$-space with the same moment polytope) is equipped 
with an intrinsic $T^{n}$-invariant complex structure compatible with the intrinsic symplectic form. In other words, $X_{\Delta}$ has an intrinsic Kähler metric. In [6] Guillemin gives an explicit formula for this Kähler metric in terms of moment data alone. The details are as follows.

Let $(X, \omega)$ be an effective Hamiltonian $T^{n}$-space, with moment map $\phi$ : $X \rightarrow \mathbb{R}^{n}$ and moment polytope $\Delta=\phi(X) \subset \mathbb{R}^{n} . \Delta$ can be described by a set of inequalities of the form $\left\langle y, u_{i}\right\rangle \geq \lambda_{i}, i=1, \ldots, d$, each $u_{i}$ being a primitive element of the lattice $\mathbb{Z}^{n} \subset \mathbb{R}^{n}$ and inward-pointing normal to the $i$ th $(n-1)$ dimensional face of $\Delta$. Let

$$
\begin{aligned}
l_{i}(y) & =\left\langle y, u_{i}\right\rangle-\lambda_{i}, i=1, \ldots, d, \\
l_{\infty}(y) & =\sum_{i=1}^{d}\left\langle y, u_{i}\right\rangle
\end{aligned}
$$

and $\Delta^{\circ}=$ interior of $\Delta$. Then $y \in \Delta^{\circ}$ if and only if $l_{i}(y)>0$ for all $i$.

Guillemin's main result in [6] is the following formula for the restriction of $\omega$ to $\phi^{-1}\left(\Delta^{\circ}\right)$ :

$$
\omega=\sqrt{-1} \partial \bar{\partial} \phi^{*}\left(\sum_{i=1}^{d} \lambda_{i}\left(\log l_{i}\right)+l_{\infty}\right) .
$$

The proof of this formula, which will be more relevant to us then the formula itself, goes as follows. For the intrinsic complex structure, $\phi^{-1}\left(\Delta^{\circ}\right)$ is the complex torus $M=\mathbb{C}^{n} / 2 \pi \sqrt{-1} \mathbb{Z}^{n}$, and $T^{n}=\mathbb{R}^{n} / 2 \pi \mathbb{Z}^{n}$ acts on $M$ by the action:

$$
T^{n} \times M \rightarrow M,(t, z) \mapsto z+\sqrt{-1} t .
$$

If $\omega$ is a $T^{n}$-invariant Kähler form on $M$, for which the $T^{n}$ action is Hamiltonian, then there exists a function $F=F(x), x=\operatorname{Re} z$, such that $\omega=2 \sqrt{-1} \partial \bar{\partial} F$ and the moment map $\phi: M \rightarrow \mathbb{R}^{n}$ is given by $\phi(z)=\partial F / \partial x$.

It follows that $\omega$ can be written in the form

$$
\frac{\sqrt{-1}}{2} \sum_{j, k=1}^{n} \frac{\partial^{2} F}{\partial x_{j} \partial x_{k}} d z_{j} \wedge d \bar{z}_{k}
$$

and the restriction to $\mathbb{R}^{n}=\left(\operatorname{Re} \mathbb{C}^{n}\right)$ of the Kähler metric is the Riemannian metric

$$
\sum_{j, k=1}^{n} \frac{\partial^{2} F}{\partial x_{j} \partial x_{k}} d x_{j} d x_{k}
$$

Guillemin shows that, under the Legendre transform given by the moment map

$$
y=\frac{\partial F}{\partial x}=\phi,
$$


this is the pull-back of the metric

$$
\sum_{j, k=1}^{n} \frac{\partial^{2} G}{\partial y_{j} \partial y_{k}} d y_{j} d y_{k}
$$

on $\Delta^{\circ}$, where

$$
G=\frac{1}{2} \sum_{k=1}^{d} l_{k}(y) \log l_{k}(y) .
$$

Moreover, the inverse of the Legendre transform (3) is

$$
x_{i}=\frac{\partial G}{\partial y_{i}}+a_{i}, i=1, \ldots, n,
$$

the $a_{i}$ being constants. This means that, up to a linear factor in $y, G$ is the Legendre function dual to $F$, i.e.

$$
F(x)=\sum_{i=1}^{n} y_{i} \frac{\partial G}{\partial y_{i}}-G(y)
$$

evaluated at $y=\partial F / \partial x$.

One can now easily deduce (1) from (2), (蛋) and (6).

Note also that it follows from (3) and (5) that the matrix

$$
\left(G_{i j}\right)=\left(\frac{\partial^{2} G}{\partial y_{i} \partial y_{j}}\right)
$$

is the inverse of the matrix

$$
\left(F_{i j}\right)=\left(\frac{\partial^{2} F}{\partial x_{i} \partial x_{j}}\right),
$$

at $y=\partial F / \partial x$.

This construction of Guillemin can also be used to obtain any other toric Kähler metric on $X_{\Delta}$ from data on $\Delta$ alone. Indeed, one starts with any $G^{\prime}$ : $\Delta^{\circ} \rightarrow \mathbb{R}^{n}$ of the form

$$
G^{\prime}=\frac{1}{2}\left(\sum_{k=1}^{d} l_{k}(y) \log l_{k}(y)\right)+f(y),
$$

where $f$ is a smooth function on some open subset of $\mathbb{R}^{n}$ containing $\Delta$, such that the Hessian of $G^{\prime}$ is positive definite on $\Delta^{\circ}$. Using the above Legendre transformation process in reverse, one gets a function $F^{\prime}=F^{\prime}(x)$ on $M=\mathbb{C}^{n} / 2 \pi \sqrt{-1} \mathbb{Z}^{n}$ that is the Kähler potential for a new Kähler metric $\omega^{\prime}=2 \sqrt{-1} \partial \bar{\partial} F^{\prime}$. Because the behaviour of $\omega^{\prime}$ at infinity is the same as that of $\omega$ (we only changed $G$ by a nonsingular function $f$ on $\Delta$ ), this new Kähler metric compactifies in the same

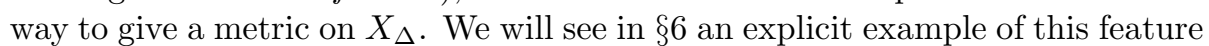
of Guillemin's construction. 
Remark 3.1 In the previous description we are looking at $X_{\Delta}$ as a fixed complex manifold $\left(X_{\Delta}, J\right)$ with an holomorphic $T^{n}$-action, having two different invariant Kähler forms, the canonical $\omega$ and the new $\omega^{\prime}$, for which the action is Hamiltonian. Since, by construction, the moment polytopes for $\left(X_{\Delta}, \omega\right)$ and $\left(X_{\Delta}, \omega^{\prime}\right)$ are the same $\Delta \subset \mathbb{R}^{n}$, we know from Delzant's Theorem 2.2 that there is an equivariant symplectomorphism $\psi:\left(X_{\Delta}, \omega\right) \rightarrow\left(X_{\Delta}, \omega^{\prime}\right)$. Using $\psi$ we can view $X_{\Delta}$ as a fixed symplectic manifold $\left(X_{\Delta}, \omega\right)$ with a Hamiltonian $T^{n}$-action, having two different invariant compatible complex structures, the canonical $J$ and a new $J^{\prime}=\psi^{*}(J)$.

This two points of view are completely equivalent, the later being perhaps more natural in terms of symplectic geometry, while the former is standard in Kähler geometry and more convenient for computational purposes.

\section{Scalar curvature and the extremal condition}

Denote by $\left(G^{i j}\right)$ and $\left(F^{i j}\right)$ the inverses of the matrices $\left(G_{i j}\right)$ and $\left(F_{i j}\right)$ defined by (7) and (8), with $G$ given by the right-hand side of (9). Recall that $G_{i j}=F^{i j}$ at $y=\partial F / \partial x$. Denote by $\operatorname{det} G$ and $\operatorname{det} F$ the determinants of the matrices $\left(G_{i j}\right)$ and $\left(F_{i j}\right)$. Throughout this section repeated indices will be summed from 1 to $n$.

The scalar curvature $R$ of the Kähler metric (2) is given by

$$
R=-\frac{1}{2} F^{i j} \frac{\partial^{2} \log (\operatorname{det} F)}{\partial x_{i} \partial x_{j}}
$$

(this follows from the standard formulas for the scalar curvature of a Kähler metric, see for example [1] , using the fact that in our case the Kähler potential $F$ only depends on the real part of the complex coordinate $z$ ). To express $R$ as a function on the image of the moment map $\phi=\partial F / \partial x$, i.e. as a function of $y$, we first note that under the change of coordinates $y=\partial F / \partial x$ we have (using (5))

$$
F^{i j} \frac{\partial}{\partial x_{j}}=\frac{\partial^{2} G}{\partial y_{i} \partial y_{j}} \frac{\partial}{\partial x_{j}}=\frac{\partial x_{j}}{\partial y_{i}} \frac{\partial}{\partial x_{j}}=\frac{\partial}{\partial y_{i}} .
$$

From (10) using (11) we then have that

$$
\begin{aligned}
2 R & =-F^{i j} \frac{\partial^{2} \log (\operatorname{det} F)}{\partial x_{i} \partial x_{j}}=-\frac{\partial}{\partial y_{j}} \frac{\partial \log (\operatorname{det} F)}{\partial x_{j}} \\
& =\frac{\partial}{\partial y_{j}} \frac{\partial \log (\operatorname{det} G)}{\partial x_{j}}=\frac{\partial}{\partial y_{j}}\left(\frac{\partial \log (\operatorname{det} G)}{\partial y_{i}} \frac{\partial y_{i}}{\partial x_{j}}\right) \\
& =\frac{\partial}{\partial y_{j}}\left(\frac{\partial \log (\operatorname{det} G)}{\partial y_{i}} F_{i j}\right)=\frac{\partial}{\partial y_{j}}\left(G^{i j} \frac{\partial \log (\operatorname{det} G)}{\partial y_{i}}\right) \\
\Rightarrow 2 R & =\frac{\partial}{\partial y_{j}}\left(G^{i j} \frac{\partial \log (\operatorname{det} G)}{\partial y_{i}}\right) .
\end{aligned}
$$


Formula (12) can be simplified using the fact that if $U=\left(u^{a b}\right)$ and $Y=\left(y^{a b}\right)$ are symmetric matrices, with $Y$ positive definite, then

$$
u^{a b} \frac{\partial}{\partial y^{a b}}(\log (\operatorname{det} Y))=\operatorname{trace}\left(U Y^{-1}\right) .
$$

Indeed, from (12) using (13), we then have

$$
\begin{aligned}
2 R & =\frac{\partial}{\partial y_{i}}\left(G^{i j} \frac{\partial \log (\operatorname{det} G)}{\partial y_{j}}\right) \\
& =\frac{\partial}{\partial y_{i}}\left(G^{i j} \frac{\partial \log (\operatorname{det} G)}{\partial G_{a b}} \frac{\partial G_{a b}}{\partial y_{j}}\right) \\
& =\frac{\partial}{\partial y_{i}}\left(G^{i j} \frac{\partial G_{a b}}{\partial y_{j}} G^{a b}\right)=\frac{\partial}{\partial y_{i}}\left(G^{i j} \frac{G_{j b}}{\partial y_{a}} G^{a b}\right) \\
& =-\frac{\partial}{\partial y_{i}}\left(\frac{\partial G^{i j}}{\partial y_{a}} G_{j b} G^{a b}\right)=-\frac{\partial}{\partial y_{i}}\left(\frac{\partial G^{i j}}{\partial y_{a}} \delta_{j}^{a}\right) \\
& =-\frac{\partial^{2} G^{i j}}{\partial y_{i} \partial y_{j}} .
\end{aligned}
$$

The Euler-Lagrange equation defining an extremal Kähler metric is given by

$$
(\operatorname{grad} R)^{(1,0)}=\left(F^{i j} \frac{\partial R}{\partial x_{j}}\right) \frac{\partial}{\partial z_{i}} \equiv \text { holomorphic vector field . }
$$

Because the coefficients of the $\partial / \partial z_{i}$ are real, the only way the above vector field is holomorphic is if

$$
F^{i j} \frac{\partial R}{\partial x_{j}}=\frac{\partial R}{\partial y_{i}} \equiv \text { constant }, i=1, \ldots, n .
$$

We summarize the previous calculations in the following

Theorem 4.1 Let $\Delta \subset \mathbb{R}^{n}$ be a Delzant polytope defined by the inequalities $\left\langle y, u_{i}\right\rangle \geq \lambda_{i}, i=1, \ldots, d$, each $u_{i}$ being a primitive element of the lattice $\mathbb{Z}^{n} \subset$ $\mathbb{R}^{n}$ and inward-pointing normal to the $i$ th $(n-1)$-dimensional face of $\Delta$. Let $l_{i}(y)=\left\langle y, u_{i}\right\rangle-\lambda_{i}$, and define $G$ by the right-hand side of (9) and $\left(G_{i j}\right)$ by (1). Let $F$ be the Legendre function dual to $G$ and $\left(G^{i j}\right)=\left(G_{i j}\right)^{-1}$. Then

(i) the scalar curvature of the Kähler metric on $X_{\Delta}$ defined by (2) is given by

$$
R=-\frac{1}{2} \sum_{i, j=1}^{n} \frac{\partial^{2} G^{i j}}{\partial y_{i} \partial y_{j}} ;
$$

(ii) this Kähler metric is extremal if and only if

$$
\frac{\partial R}{\partial y_{i}} \equiv \text { constant }, i=1, \ldots, n,
$$

i.e., $R$ is an affine function of $y$. 
Example 4.2 For $\mathbb{C} P^{2}$ with its standard Fubini-Study Kähler metric, the linear functions defining the moment polytope (an equilateral right triangle) are:

$$
l_{1}(y)=y_{1}, l_{2}(y)=y_{2}, l_{3}(y)=1-y_{1}-y_{2} .
$$

We then have

$$
\begin{gathered}
G=\frac{1}{2}\left(y_{1} \log y_{1}+y_{2} \log y_{2}+\left(1-y_{1}-y_{2}\right) \log \left(1-y_{1}-y_{2}\right)\right), \\
\left(G_{i j}\right)=\frac{1}{2\left(1-y_{1}-y_{2}\right)}\left(\begin{array}{cc}
\frac{1-y_{2}}{y_{1}} & 1 \\
1 & \frac{1-y_{1}}{y_{2}}
\end{array}\right), \\
\left(G^{i j}\right)=2\left(\begin{array}{cc}
y_{1}\left(1-y_{1}\right) & -y_{1} y_{2} \\
-y_{1} y_{2} & y_{2}\left(1-y_{2}\right)
\end{array}\right)
\end{gathered}
$$

and $R=2+1+1+2=6$.

\section{A combinatorial formula}

It is well known that on any Kähler manifold $X$ the total scalar curvature is a topological invariant, depending only on the Kähler class $\Omega$ represented by the metric $\omega$ and the first Chern class $c_{1}(X)$ of the underlying complex manifold. More precisely we have that

$$
\bar{R}=\int_{X} R_{\omega} d v_{\omega}=\frac{2 \pi}{(n-1) !} \int_{X} c_{1}(X) \wedge \omega^{n-1}=\frac{2 \pi}{(n-1) !} c_{1}(X) \wedge \Omega^{n-1} .
$$

For a toric variety $X_{\Delta}$, associated to a Delzant polytope $\Delta \subset \mathbb{R}^{n}$, the righthand side of the above equation can be computed in very simple terms from combinatorial data on $\Delta$. Recall that $\Delta$ is defined by the system of inequalities $\left\langle y, u_{i}\right\rangle \geq \lambda_{i}, i=1, \ldots, d$, each $u_{i}$ being a primitive element of the lattice $\mathbb{Z}^{n} \subset \mathbb{R}^{n}$ and inward-pointing normal to the $i$ th $(n-1)$-dimensional face of $\Delta$. Let $v_{i}=-u_{i}$, the outward-pointing normal to this face, and let $s_{i}=-\lambda_{i}$. Then $\Delta$ can also be defined by the inequalities:

$$
\left\langle y, v_{i}\right\rangle \leq s_{i}, i=1, \ldots, d .
$$

Let $v\left(s_{1}, \ldots, s_{d}\right)$ denote the Euclidean volume of this set.

As before, define $l_{i}(y)=\left\langle y, u_{i}\right\rangle-\lambda_{i}=s_{i}-\left\langle y, v_{i}\right\rangle, i=1, \ldots, d$, and let $\phi: X_{\Delta} \rightarrow \Delta \subset \mathbb{R}^{n}$ be the moment map. The subset $X_{i} \subset X_{\Delta}$ defined by $l_{i} \circ \phi=0$ is a complex submanifold of real codimension 2. It is the pre-image in $X$ of the $i$ th $(n-1)$-dimensional face of $\Delta$. Let $c_{i}$ be the cohomology class in $H^{2}\left(X_{\Delta}, \mathbb{Z}\right)$ dual to the homology class $\left[X_{i}\right]$ in $H_{2 n-2}\left(X_{\Delta}, \mathbb{Z}\right)$.

We then have that (see [6] and [3])

$$
\frac{\Omega}{2 \pi}=\frac{[\omega]}{2 \pi}=\sum_{i=1}^{d} s_{i} c_{i}
$$


and

$$
c_{1}\left(X_{\Delta}\right)=\sum_{i=1}^{d} c_{i} .
$$

Moreover, it follows from (2) and the change of coordinates (3) that

$$
\begin{aligned}
& \int_{X_{\Delta}} \exp (\omega)=\int_{X_{\Delta}} \frac{\omega^{n}}{n !}=(2 \pi)^{n} \operatorname{Volume}(\Delta) \\
\Rightarrow & v\left(s_{1}, \ldots, s_{d}\right)=\int_{X_{\Delta}} \exp \left(\sum s_{i} c_{i}\right) \\
\Rightarrow & \sum_{i=1}^{d} \frac{\partial v}{\partial s_{i}}=\int_{X_{\Delta}}\left(\sum c_{i}\right) \exp \left(\sum s_{i} c_{i}\right)
\end{aligned}
$$

Using (16) and (17) we get

$$
\frac{2 \pi}{(n-1) !} c_{1}\left(X_{\Delta}\right) \wedge \Omega^{n-1}=(2 \pi)^{n} \sum_{i=1}^{d} \frac{\partial v}{\partial s_{i}},
$$

the desired combinatorial formula for the right-hand side of (15)).

Regarding its left-hand side, using again (2) and (3), and noting that the scalar curvature $R$, as a function on $\phi^{-1}\left(\Delta^{\circ}\right)=\mathbb{C}^{n} / 2 \pi \sqrt{-1} \mathbb{Z}^{n}$, depends only on $x=\operatorname{Re} z$, we have that

$$
\int_{X_{\Delta}} R d v=\int_{X_{\Delta}} R \exp (\omega)=(2 \pi)^{n} \int_{\Delta} R(y) d y .
$$

Taking into account (14), we have proved the following

Proposition 5.1 Let $\Delta=\Delta\left(s_{1}, \ldots, s_{d}\right) \subset \mathbb{R}^{n}$ be a Delzant polytope defined by the inequalities $\left\langle y, v_{i}\right\rangle \leq s_{i}, i=1, \ldots, d$, each $v_{i}$ being a primitive element of the lattice $\mathbb{Z}^{n} \subset \mathbb{R}^{n}$ and outward-pointing normal to the ith $(n-1)$-dimensional face of $\Delta$. Let $l_{i}(y)=s_{i}-\left\langle y, v_{i}\right\rangle$, and define $G$ by the right-hand side of (9) and $\left(G_{i j}\right)$ by (1). Let $\left(G^{i j}\right)=\left(G_{i j}\right)^{-1}$ and $v\left(s_{1}, \ldots, s_{d}\right)$ be the Euclidean volume of $\Delta\left(s_{1}, \ldots, s_{d}\right)$. Then

$$
\sum_{i=1}^{d} \frac{\partial v}{\partial s_{i}}=-\frac{1}{2} \int_{\Delta}\left(\sum_{i, j} \frac{\partial^{2} G^{i j}}{\partial y_{i} \partial y_{j}}\right) d y .
$$

It would be interesting to know if $(18)$ is more than a curiosity, possibly having some useful application in particular when $G$ has the canonical form given by (化). 


\section{Calabi's family of extremal metrics on $\mathbf{C} P^{2} \sharp \overline{\mathbf{C P}}^{2}$}

In [1] Calabi constructed families of extremal metrics on holomorphic $\mathbb{C} P^{1}$ bundles over $\mathbb{C} P^{n}$, parametrized by the cohomology classes of the corresponding Kähler forms. When these bundles are holomorphically non-trivial the corresponding group of complex automorphisms is not reductive, and so, due to a theorem of Matsushima [8] and Lichnérowicz [7], we know apriori that these metrics have non-constant scalar curvature. The purpose of this section is to recast Calabi's simplest example, $\mathbb{C} P^{2} \sharp \overline{\mathbb{C} P}^{2}$, in the language of the previous sections. For yet another approach to Calabi's families of extremal metrics see $[9]$.

The linear functions defining the moment polytope are now:

$$
l_{1}(y)=y_{1}, l_{2}(y)=y_{2}, l_{3}(y)=1-y_{1}-y_{2}, l_{4}(y)=y_{1}+y_{2}-a,
$$

where $0<a<1$ parametrizes the amount by which we are blowing up the standard $\mathbb{C} P^{2}$. It is easy to check that the metric corresponding, under Guillemin's construction, to this linear functions is not extremal. As explained at the end of \$, in order to get an extremal metric within the same Kähler class we can try to change this one by adding to the corresponding $G$ a smooth function on the corresponding $\Delta$. The fact that the maximal compact subgroup of the group of complex automorphisms of $\mathbb{C} P^{2} \sharp \overline{\mathbb{C}}^{2}$ is $U(2)$, and not just $T^{2}$, translates into the fact that the smooth function we are looking for will only depend on the variable $\psi=y_{1}+y_{2}$. Hence we will consider $G$ of the form:

$$
G=\frac{1}{2}\left(\sum_{i=1}^{4} l_{i}(y) \log l_{i}(y)+f(\psi)\right)
$$

Calabi's construction is on $\mathbb{C}^{2} \backslash\{0\}$, say with coordinates $w=\left(w_{1}, w_{2}\right)$. His $U(2)$-invariant extremal Kähler metric is generated by a Kähler potential $\Phi(w, \bar{w})=u(t), t=\log \left(\left|w_{1}\right|^{2}+\left|w_{2}\right|^{2}\right)$, where $u: \mathbb{R} \rightarrow \mathbb{R}$ is a smooth function satisfying

$$
u^{\prime}(t)>0, u^{\prime \prime}(t)>0
$$

and

$$
a=\lim _{t \rightarrow-\infty} u^{\prime}(t), 1=\lim _{t \rightarrow+\infty} u^{\prime}(t)
$$

The scalar curvature is given by

$$
R=12 c_{1} u^{\prime}(t)+6 c_{2}
$$

where $c_{1}$ and $c_{2}$ are constants given by

$$
c_{1}=\frac{2 a}{(1-a)\left(1+4 a+a^{2}\right)} \text { and } \quad c_{2}=\frac{1-3 a^{2}}{(1-a)\left(1+4 a+a^{2}\right)} \text {. }
$$


On $\Delta$ we know from Theorem 4.1 (ii) that $R$ is an affine function of $\psi=y_{1}+y_{2}$, i.e. $u^{\prime}(t)=\alpha \psi+\beta$ for some $\alpha, \beta \in \mathbb{R}$. Because of the "boundary conditions" (21) we actually have that

$$
u^{\prime}(t)=\psi=y_{1}+y_{2} .
$$

Equation (22), together with the non-degeneracy condition (20), gives an implicit formula for $t$ as a function of $\psi$. The extremal condition in Calabi's construction is then given by

$$
\frac{d t}{d \psi}=\frac{1-a}{(\psi-a)(1-\psi)}+\frac{2 a(1-a)}{2 a \psi^{2}+\left(1+2 a-a^{2}\right) \psi+2 a^{2}} .
$$

We are now in position to determine $f(\psi)$. From (6) and (19) we have that

$$
\begin{aligned}
\frac{1}{2} u(t) & =\sum_{i=1}^{n} y_{i} \frac{\partial G}{\partial y_{i}}-G(y) \\
& =\frac{1}{2}\left(\psi+\psi f^{\prime}(\psi)-f(\psi)-\log (1-\psi)+a \log (\psi-a)\right) .
\end{aligned}
$$

Taking the derivative with respect to $\psi$ and using (22) and (23) gives

$\psi\left(\frac{1-a}{(\psi-a)(1-\psi)}+\frac{2 a(1-a)}{2 a \psi^{2}+\left(1+2 a-a^{2}\right) \psi+2 a^{2}}\right)=1+\psi f^{\prime \prime}(\psi)+\frac{(1-a) \psi}{(1-\psi)(\psi-a)}$

which immediately implies

$$
f^{\prime \prime}(\psi)=\frac{2 a(1-a)}{2 a \psi^{2}+\left(1+2 a-a^{2}\right) \psi+2 a^{2}}-\frac{1}{\psi},
$$

a formula that can be explicitly integrated twice. It is now elementary, though tedious, to verify that using (19), (24) and (14) one indeed gets

$$
R=12 c_{1} \psi+6 c_{2} .
$$




\section{References}

[1] E.Calabi, Extremal Kähler metrics, in Seminar on Differential Geometry, ed. S.T.Yau, Annals of Math. Studies 102, 159-290, Princeton Univ. Press, 1982.

[2] E.Calabi, Extremal Kähler metrics II, in Differential Geometry and Complex Analysis, eds. I.Chavel and H.M.Farkas, Springer-Verlag, 95-114, 1985.

[3] V.I.Danilov, The geometry of toric varieties, Russian Math. Surveys $\mathbf{3 3}$ (1978), 97-154.

[4] T.Delzant, Hamiltoniens périodiques et image convex de l'application moment, Bull. Soc. Math. France, 116 (1988), 315-339.

[5] V.Guillemin, Moment Maps and Combinatorial Invariants of Hamiltonian $T^{n}$-spaces, Progress in Math. 122, Birkhäuser, 1994.

[6] V.Guillemin, Kähler structures on toric varieties, Journal of Differential Geometry 40 (1994), 285-309.

[7] A.Lichnérowicz, Sur les transformations analytiques des variéties kählériennes, C. R. Acad. Sci. Paris 244 (1957), 3011-3014.

[8] Y.Matsushima, Sur la structure du groupe d'homéomorphismes analytiques d'une certaine variété kählérienne, Nagoya Math. Journal 11 (1957), 145150 .

[9] S.Simanca, A note on extremal metrics of non-constant scalar curvature, Israel Journal Math. 78 (1992), 85-93. 